\title{
A four component picture for jet induced correlations in heavy ion collisions?
}

\author{
Recent results on two and three particle correlations
}

\author{
Anne Sickles ${ }^{\mathrm{a}}$ \\ Brookhaven National Laboratory, Upton, NY 11973, USA
}

Received: 14 September 2008 / Published online: 9 January 2009

(C) Springer-Verlag / Società Italiana di Fisica 2008

\begin{abstract}
These proceedings review recent results from two and three particle correlations in heavy ion collisions. In particular we discuss the modified structure of the away side jet correlations. Under the assumption that the away side can be decomposed into a punch through component at $\Delta \phi=\pi$ and a shoulder component with a peak displaced from $\pi$ many similar properties are observed between the ridge and shoulder. The particle ratios, yields and $p_{T}$ spectra are in near agreement. We also highlight important future measurements, including investigating if the decomposition of the away side jet correlations remains reasonable with high $p_{T}$ triggers and technical improvements to the extraction of jet induced correlations.
\end{abstract}

\section{Introduction}

The main goal of heavy ion collisions is to determine the properties of the hot dense matter created in central collisions. While there are many observables of interest, jets are very powerful because they are produced only in the initial state. The scattered partons then interact with the produced matter from its early stage until they leave the collision region. This sensitivity to the time evolution of the system and that the initial properties of the jets can be studied in small systems, $p+p$ and $d+A u$, provide the ability to study properties of the matter itself and how it hadronizes.

Single particle measurements of jet induced particle have provided constraints on opacity of the matter [1], but multiparticle correlation measurements provide tomographic information and test the consistency of these models. Additionally they are also sensitive to changes in the jet fragmentation process suggested by single particle results showing a

a e-mail: anne@bnl.gov dramatic change in the particle ratios from small systems to central $\mathrm{Au}+\mathrm{Au}[2,3]$ for $p_{T}<5 \mathrm{GeV} / \mathrm{c}$.

Here we describe some of the recent results of jet correlations in heavy ion collisions.

\section{Jet landscape in heavy ion collisions}

The two particle correlation method has been described in detail before (for example, see Ref. [4]). In $p+p$ collisions hard scattering results in two jets approximately back-toback in azimuth. In two-particle azimuthal correlations this results in an excess of particles at small $\Delta \phi$ and at $\Delta \phi=\pi$ in what are termed the near and away side jets, respectively. These correlations are localized in the $\Delta \eta$ direction as well, however the di-jets are not constrained to be back to back in $\eta$ because the center of mass of the parton-parton scattering is not necessarily the collision center of mass. One of the most striking results from RHIC is that this qualitative picture is dramatically changed in central collisions at RHIC; two new structures are observed. There is a peak on the away side displaced from $\Delta \phi=\pi$ by approximately one radian, the shoulder (see Fig. 2.1) and an elongated structure in $\Delta \eta$ on the near side, the ridge (see Fig. 2.2). The origins and properties of these structures are under investigation and for the purposes of these proceedings we will treat the observed jet correlations in this four component picture: the near side jet (small $\Delta \phi$, small $\Delta \eta$ ); the ridge (small $\Delta \phi$, wide in $\Delta \eta)$; the away side punch through jet $(\Delta \phi \approx \pi)$; and the shoulder $(\Delta \phi \approx \pi \pm 1)$. It is still a matter of experimental investigation to what extent the shoulder and punch through distinction is the right picture. However, by making this separation and looking at the properties of the extracted shoulder suggestive similarities are seen to the properties of the ridge. 


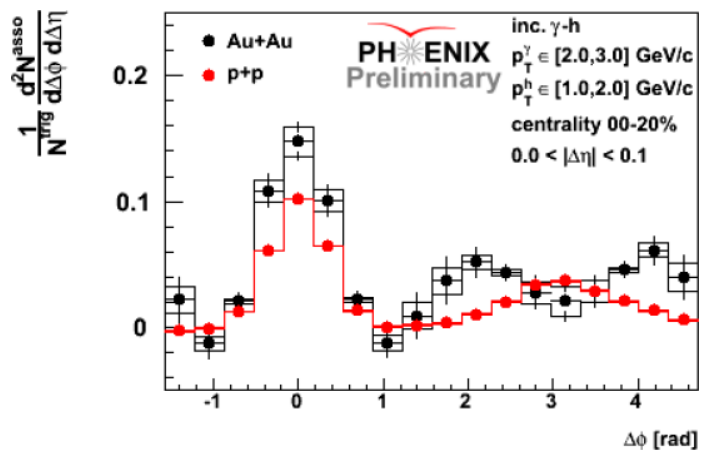

Fig. 2.1 $\Delta \phi$ distributions of correlated inclusive photon-hadron pairs in central $\mathrm{Au}+\mathrm{Au}$ collisions (black) and $\mathrm{p}+\mathrm{p}$ collisions (red). The photons have $2.0<p_{T}<3.0 \mathrm{GeV} / \mathrm{c}$ and the hadrons have $1.0<p_{T}<2.0 \mathrm{GeV} / \mathrm{c}$. From Ref. [5]

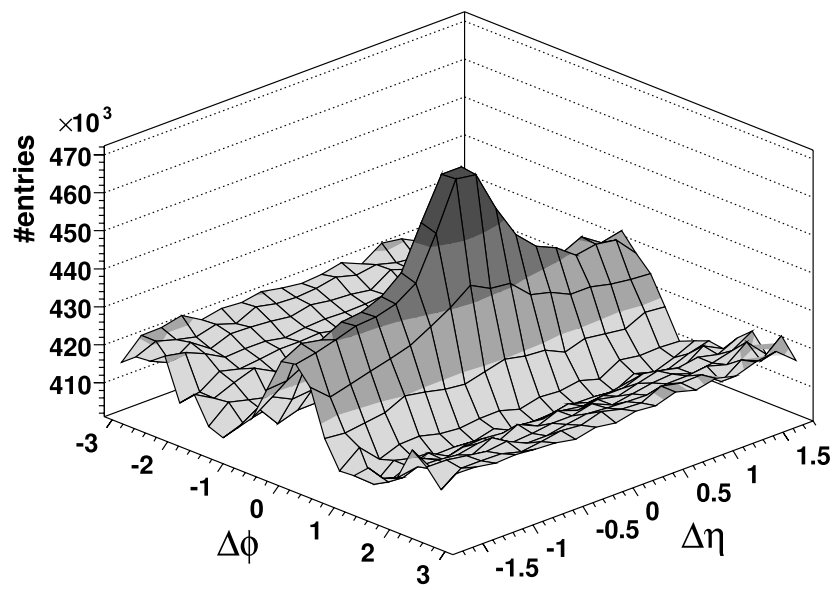

Fig. 2.2 $\Delta \phi$ v. $\Delta \eta$ distributions of correlated hadron pairs in central $\mathrm{Au}+\mathrm{Au}$ collisions. Triggers have $3.0<p_{T}<4.0 \mathrm{GeV} / \mathrm{c}$ and associated particles have $2.0<p_{T}<p_{T, \text { trig. From Ref. [6] }}$

\subsection{The ridge}

Extraction of the ridge The ridge yield is extracted by measuring small $\Delta \phi$ correlations in a $\Delta \eta$ region that is expected to have only a small yield from jets. Jana Bielcikova spoke at this conference [7] about what is known experimentally about the ridge in heavy ion collisions as well as theoretical ideas about its origin. The ridge is extended in $\Delta \eta$; currently there are no measurements of its full extent; PHOBOS sees evidence for a ridge out to $\Delta \eta=4$ [8], the end of their acceptance in correlations between trigger hadrons with $p_{T}>2.5 \mathrm{GeV} / \mathrm{c}$ and partner hadrons with $p_{T}>20 \mathrm{MeV} / \mathrm{c}$.

\subsection{The shoulder}

Extraction of the shoulder The PHENIX Collaboration has used a variety of techniques to extract information about the shoulder from two-particle correlations. Figure 2.3 [4]

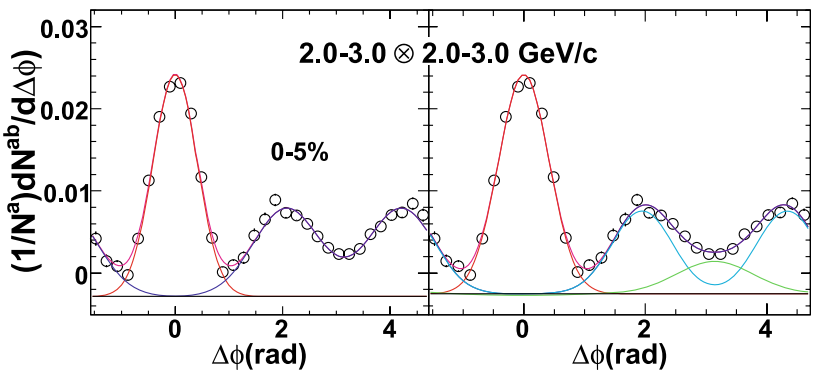

Fig. 2.3 Fits to distributions of correlated hadrons. The left panel shows the away side described by a two Gaussian fit and the right panel shows the away side described by a three Gaussian fit with the additional Gaussian centered at $\Delta \phi=\pi$. Both fits describe the data relatively well. From Ref. [4]

shows two different methods to extract the shoulder location under the assumption of a Gaussian shape. On the left is a two Gaussian fit with the away side Gaussians displaced from $\pi$ by $\pm D$ rad. On the right is a three-Gaussian fit which includes an additional Gaussian at $\Delta \phi=\pi$ corresponding to punch-through jets, those back-to-back correlations as in $\mathrm{p}+\mathrm{p}$. With both two- and three-Gaussian fits PHENIX has found that the $D$ parameter increases slightly with $p_{T}$ for central collisions, Fig. 2.4. Interestingly, no significant dependence on either collision energy or the colliding nuclei is seen either. Figure 2.5 shows $D$ for $\mathrm{Au}+\mathrm{Au}$ and $\mathrm{Cu}+\mathrm{Cu}$ collisions at both 200 and $62.4 \mathrm{GeV}$ center of mass energy extracted from a two-Gaussian fit. These results pose a challenge to identifying the shoulder structure with a Mach Cone shock-wave since the angle of the shoulder should be [10]: $\cos \theta_{M}=\frac{\bar{c}_{s}}{v_{j e t}}$ where $v_{\text {jet }}$ is the velocity of the jet (essentially 1 for light quark jets) and $\overline{c_{s}}$ is the time averaged speed of sound in the matter, which change with the initial temperature and lifetime of the system.

Three particle correlations are sensitive to correlations between particles in the shoulder. Three particle correlation results from STAR, what is observed as a shoulder in two particle correlations is actually consistent with the projection of a cone structure on the away side [11]. However the opening angle observed in the three particle correlation measurements $\theta=1.38 \pm 0.02$ (stat) \pm 0.06 (sys) [11] is significantly larger that the distance between the peaks seen in two particle correlations in a similar $p_{T}$ range [4, 9]. CERES also has three particle correlation results that show evidence of conical structure on the away side [12], but a cone angle has not been extracted.

Reaction plane dependence Figures 2.6 and 2.7 show hadron-hadron correlations where the trigger particle is also selected based on it's angle with respect to the reaction plane. For the central collisions, Fig. 2.6 panels (a) and (b), there is no difference whether the trigger is in or out of the reaction plane. This is expected because when the impact 
parameter is small the initial state is fairly symmetric. In the mid-central collisions, Fig. 2.6 panels (c) and (d) and Fig. 2.7, there is a greater variation in the central values, go-

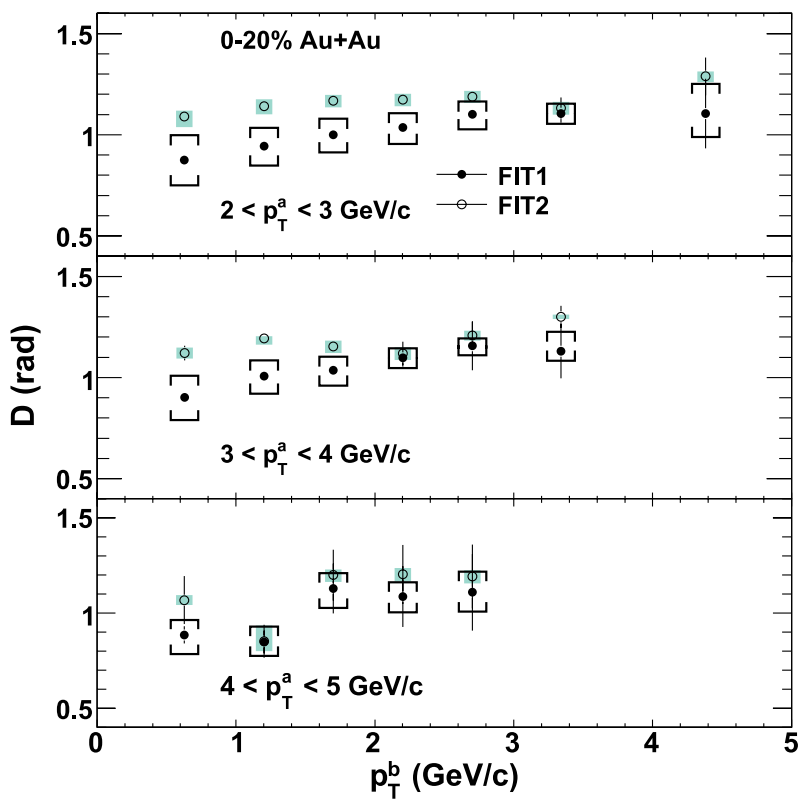

Fig. $2.4 D$ extracted with two-Gaussian (solid points) and three-Gaussian (hollow points) fit from correlations between hadron pairs in the $p_{T}$ selections indicated on the plot. From Ref. [4]

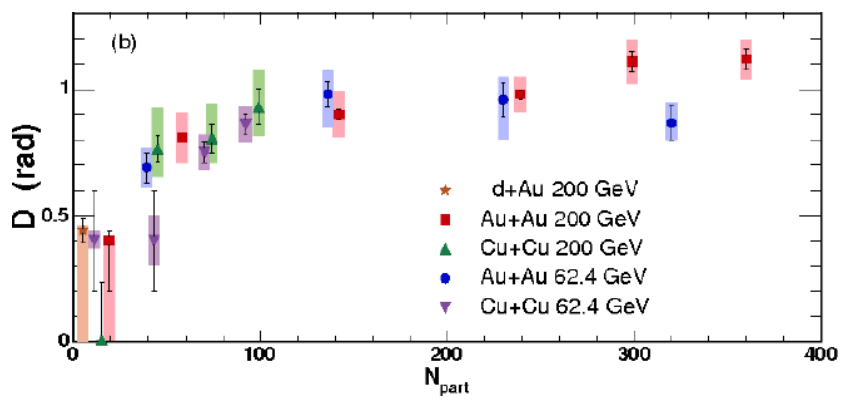

Fig. 2.5 $D$ extracted with a two-Gaussian fit from correlations between hadron pairs with triggers at $2.5<p_{T}<4.0 \mathrm{GeV} / \mathrm{c}$ and associated particles with $1.0<p_{T}<2.5 \mathrm{GeV} / \mathrm{c}$. From Ref. [9] ing from a single peak away side structure when the trigger is in plane to a double peaked away side structure when the trigger is out of plane. This is qualitatively consistent with a greater suppression of the punch through component of the away side jet when the trigger particle is out of plane as would be expected from the away side parton having a longer matter path length. However, the systematic errors from the $v_{2}$ values used in the background subtraction must be considered. As can be seen in Fig. 2.6 the effects of the $v_{2}$ error are anti-correlated between in-plane and out of plane triggers. Pushing these measurements to higher $p_{T}$ where the combinatoric background levels are smaller will help constrain these shapes. In all cases the yield in the shoulder region is nearly constant with respect to the trigger orientation with respect to the reaction plane.

\subsection{Connections between the ridge and the shoulder}

Particle ratios Figure 2.8 from STAR [14] shows the particle composition of the ridge to have a high $\frac{p+\bar{p}}{\pi^{ \pm}}$ratio, similar to $\mathrm{Au}+\mathrm{Au}$ inclusive particle ratios and greater than in the near side jet, where the inclusive particle ratio is similar to $\mathrm{p}+\mathrm{p}$ and $\mathrm{d}+\mathrm{Au}$ collisions.

PHENIX has studied the particle ratios for particles associated with a hadron trigger over the entire away side $\Delta \phi$ range and finds the ratio of $\frac{p+\bar{p}}{\pi^{ \pm}+K^{ \pm}}$to be significantly enhanced with increasing collision centrality (see Fig. 2.9) [15]. In the most central collisions, the ratios of associated particles are in agreement with those for inclusive particles, suggesting that the same hadronization mechanism could be responsible for the particles in the ridge, away side and inclusive particles. It has been speculated that as the fast parton traverses the produced matter and loses energy localized regions of increased energy density are produced [1517]. When these regions hadronize they produce particles that are correlated with the hard scattering, but have properties more similar to that of the bulk.

However, care should be taken in the interpretation of the current results. Extraction of the ridge and shoulder associated particle ratios within the same analysis is important.

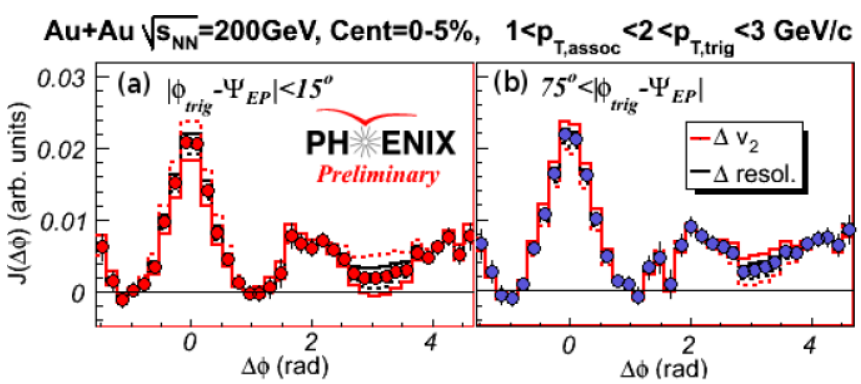

Fig. 2.6 Background subtracted distributions of hadrons with $1.0<$ $p_{T}<2.0$ associated with trigger hadrons with $2.0<p_{T}<3.0 \mathrm{GeV} / \mathrm{c}$ where the trigger has been selected to be in the reaction plane (panels

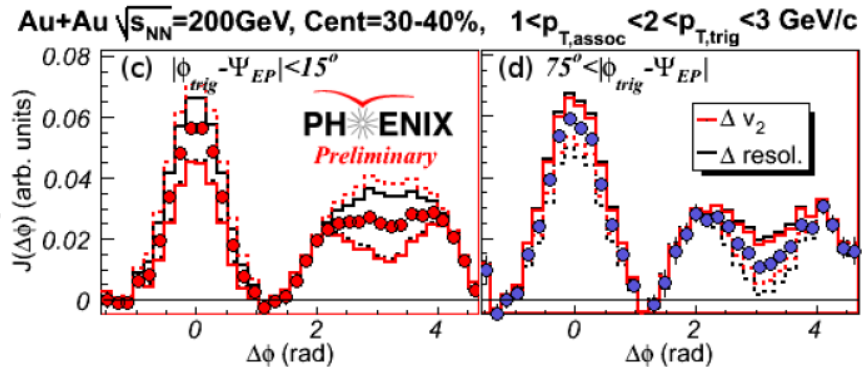

(a) and (c)) or out of the reaction plane (panels (b) and (d)). Panels (a) and (b) are 0-5\% central collisions and (c) and (d) are mid-central (30-40\%) collisions. From Ref. [13] 


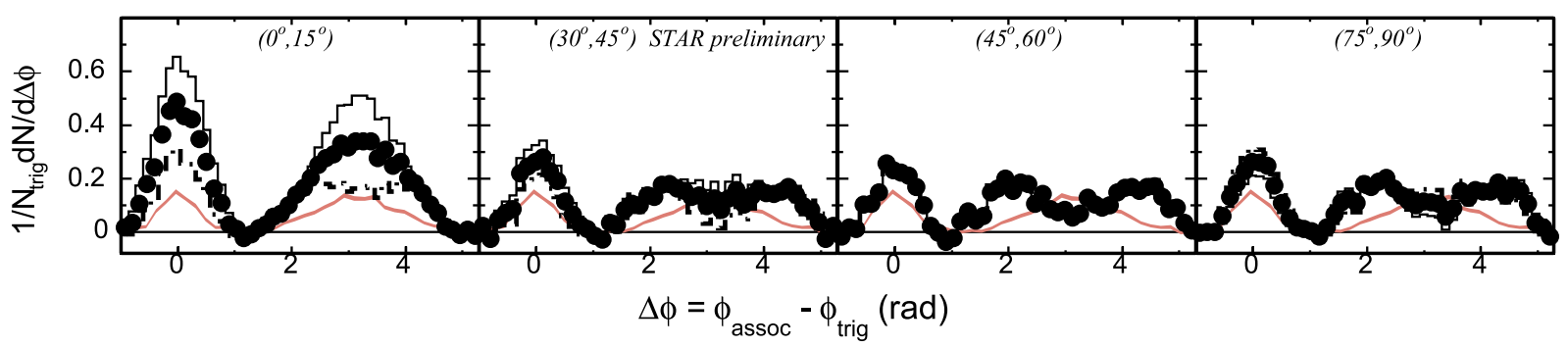

Fig. 2.7 Background subtracted distributions of hadrons with $1.0<$ $p_{T}<1.5 \mathrm{GeV} / \mathrm{c}$ associated with trigger hadrons with $3.0<p_{T}<$ $4.0 \mathrm{GeV} / \mathrm{c}$ where the trigger orientation with respect to the reaction

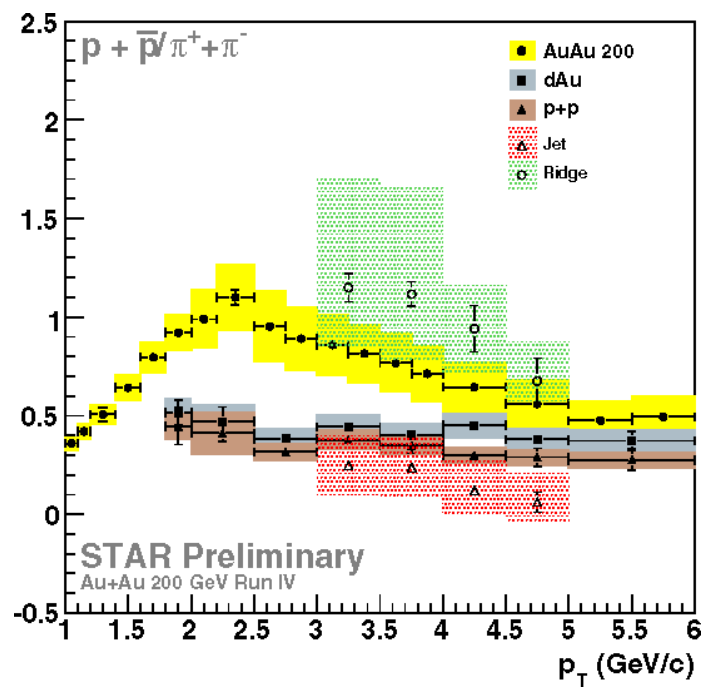

Fig. 2.8 Ratio of $(p+\bar{p}) /\left(\pi^{+}+\pi^{-}\right)$for the ridge, near side jets for $4<p_{T, \text { trig }}<6 \mathrm{GeV} / \mathrm{c}$. Inclusive particle ratios from $\mathrm{Au}+\mathrm{Au}, \mathrm{d}+\mathrm{Au}$ and $\mathrm{p}+\mathrm{p}$ collisions are shown for comparison. From Ref. [14]

While both the ridge and shoulder ratios in Figs. 2.8 and 2.9 appear consistent with the inclusive ratio reference, the associated particle ratios themselves cannot be compared directly. The PHENIX measurement includes $K^{ \pm}$in the denominator and is for both the shoulder and the away side jet region. Additionally the centrality selections and the trigger hadron $p_{T}$ ranges are different. Eliminating these differences will allow for a clean comparison.

Figure 2.10 [18] shows yields from correlations where both particles have been identified as baryons or mesons. Both particles are inside the PHENIX $\eta$ acceptance $|\eta|<$ 0.35 and the ridge and jet components have not be separated. The meson-meson near side yields increase with centrality, qualitatively consistent with an increasing ridge component in addition to the same side jet. The near side baryon-meson yields also increase with centrality up to $N_{\text {part }} \approx 250$, but then decrease for the most central collisions. This might occur if the baryons originate dominately from the ridge and the near side jet correlation is reduced, possibly because it is outside the experimental $\Delta \eta$ acceptance. Interestingly, for plane has been chosen to be within the angular window indicated on the panel. The $\mathrm{Au}+\mathrm{Au}$ events have centrality from $20-60 \%$. From Ref. [14]

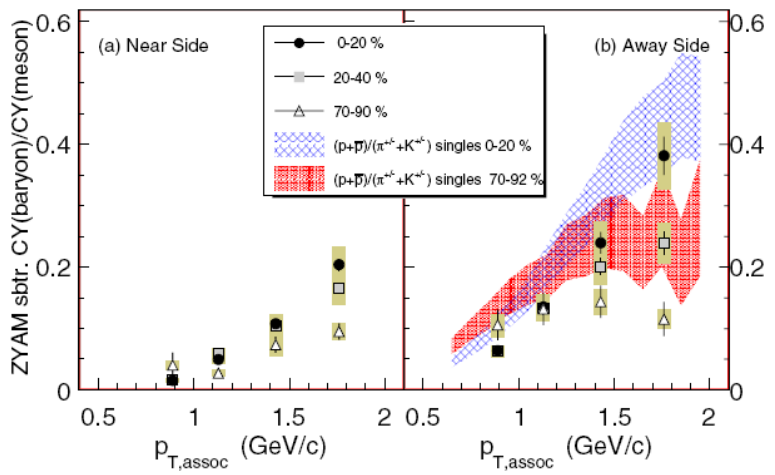

Fig. 2.9 Ratio of $(p+\bar{p}) /\left(\pi^{ \pm}+K^{ \pm}\right)$for particles associated with a hadron trigger at $2.5<p_{T}<4.0 \mathrm{GeV} / \mathrm{c}$. The left panel shows the ratio for the near side correlations (the ridge has not been subtracted and the right for the entire away side region including both the shoulder and punch-through jet region. The shaded regions show the same ratios extracted from single particle spectra in the centrality ranges shown. From Ref. [15]

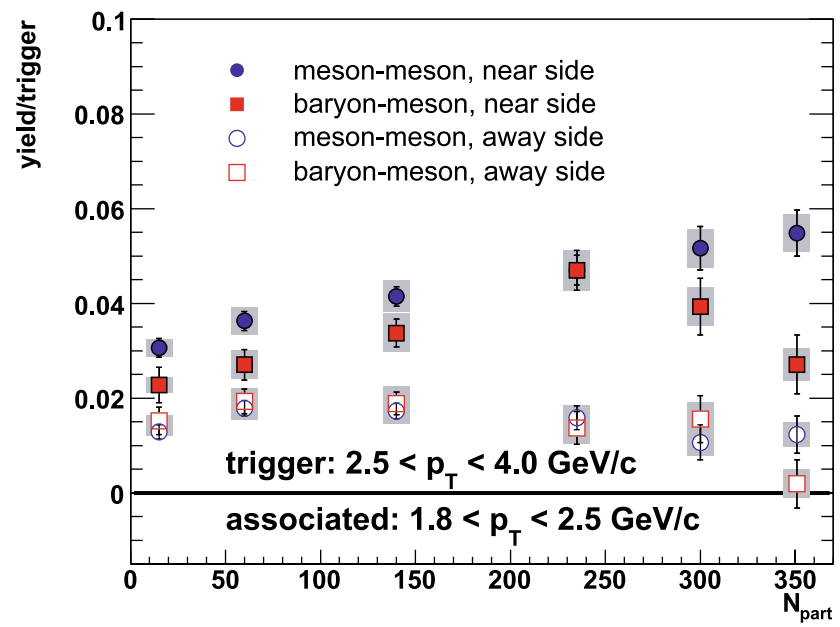

Fig. 2.10 Yield per trigger on the near, $\Delta \phi<0.94 \mathrm{rad}$ (solid points) and away $\Delta \phi>2.2 \mathrm{rad}$ (hollow points) side for baryon-meson (squares) and meson-meson (circles) correlations as a function of $N_{\text {part }}$. Triggers have $2.5<p_{T}<4.0 \mathrm{GeV} / \mathrm{c}$ and associated particles have $1.8<p_{T}<2.5 \mathrm{GeV} / \mathrm{c}$. Error bars are statistical errors and the shaded boxes show the systematic errors. There is a $13.6 \%$ normalization error which moves all points together. From Ref. [18] 
the away side (here with $|\pi-\Delta \phi|<0.94 \mathrm{rad}$ ) no dependence is seen on whether the trigger particle is a baryon or a meson.

Centrality dependence PHENIX using a three-Gaussian fitting method and inclusive photon trigger (which at moderate $p_{T}$ primarily come from $\pi^{0}$ decay where the trigger photon carries most of the $\pi^{0} p_{T}$ ) has extracted the yield per trigger, per $\Delta \eta$ for both the ridge and the shoulder, Fig. 2.11. The yields for both the ridge and the shoulder increase from peripheral to central collisions. The magnitudes of the yields are consistent at all centralities except perhaps the most central collisions.

$p_{T}$ spectra Also within the same fitting analysis, Fig. 2.12 shows the slope of the associated $p_{T}$ spectra as a function of $N_{\text {part }}$. Here again, within the experimental uncertainties the ridge and spectra slopes are similar. The ridge spectra are slightly harder than the shoulder spectra and both are significantly softer than jets in $\mathrm{p}+\mathrm{p}$ collisions.

Experimentally, reduced error bars, available from the data already on tape, and more measurements (for example, $\mathrm{Cu}+\mathrm{Cu}$ collisions, larger $p_{T}$ ranges, etc.) are needed to clarify the situation. Theoretically, most models only address the ridge or the shoulder; models which could possibly explain the connection between them could help clarify the relationship between the ridge and the shoulder.

The preliminary connections between the ridge and the shoulder are very interesting. Certainly more experimental studies are needed. To eliminate ambiguities both the ridge and the shoulder should be studied in the same analysis. The large $\mathrm{Au}+\mathrm{Au}$ statistics from the 2007 data set should be very powerful however given the observed away side in smaller

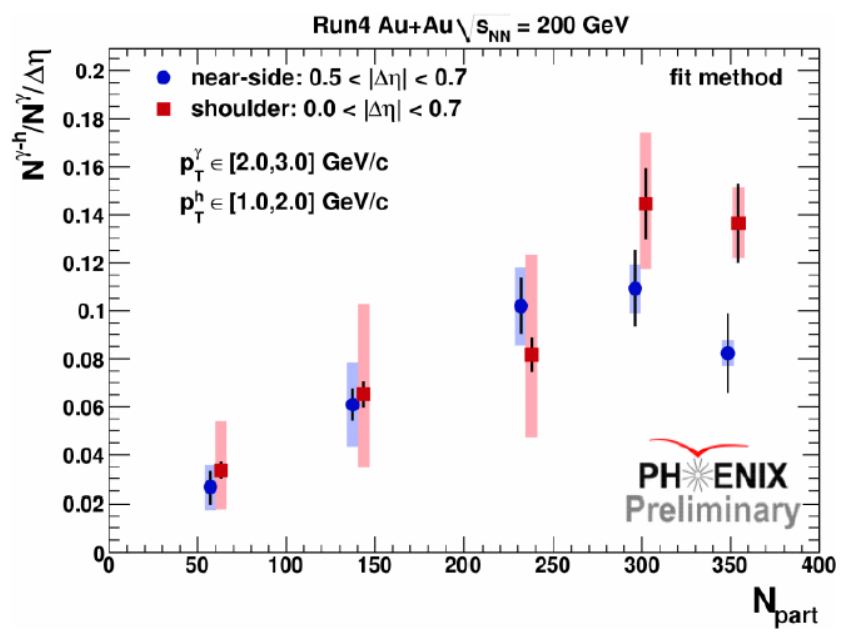

Fig. 2.11 Yield of associated hadrons per trigger per $\Delta \eta$ for both the ridge and the shoulder as a function of $N_{\text {part }}$ [5]. The triggers are inclusive photons mainly from $\pi^{0}$ decay with $2.0<p_{T}<3.0 \mathrm{GeV} / \mathrm{c}$ and the associated particles are hadrons with $1.0<p_{T}<2.0 \mathrm{GeV} / \mathrm{c}$

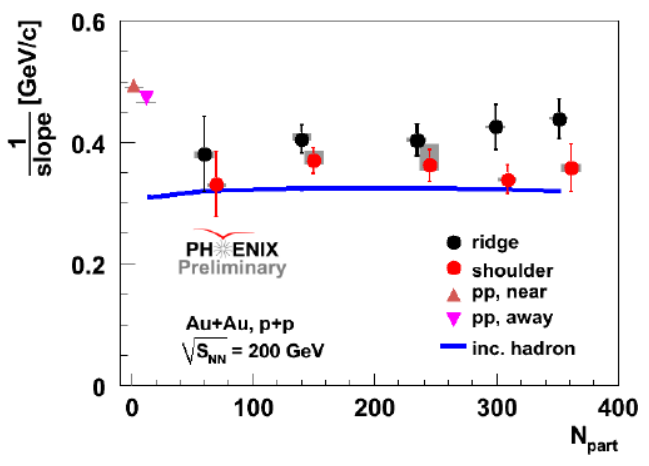

Fig. 2.12 Inverse slopes extracted from exponential fits to the $p_{T}$ spectra of hadrons with $1.0<p_{T}<5 \mathrm{GeV} / \mathrm{c}$ associated with inclusive photons with $2.0<p_{T}<3.0 \mathrm{GeV} / \mathrm{c}$ for ridge and shoulder correlations. For comparison near and away side $\mathrm{p}+\mathrm{p}$ results for the same $p_{T}$ ranges are shown along with the inclusive hadron inverse slopes. From Ref. [5]

systems and at lower energy, studies of the ridge in such systems is also important.

\section{Outlook}

\subsection{Jets at high $p_{T}$}

At high $p_{T}$ jet measurements are currently limited by statistics. Here it is less clear whether the shoulder/punch through jet distinction used above is still meaningful or whether the away side is best described as a single, perhaps widened, peak centered at $\Delta \phi=\pi$. In order to answer this question the trigger should have a high $p_{T}$ (greater than $7 \mathrm{GeV} / \mathrm{c}$ ) and be identified as a pion or direct photon to eliminate ambiguities arising from baryons. Initial work has been done in both STAR [19] and PHENIX [20, 21]. At intermediate $p_{T}$ the extraction of $D$ depends little on the fitting method used (see Fig. 2.4) since the shoulder peak is visible and the yield at $\Delta \phi=\pi$ is suppressed. However, at higher trigger $p_{T}$ the away side peak appears as a single broad peak or a flat distribution around $\Delta \phi=\pi$, so the conclusions will be more sensitive to how the parameters are extracted.

\subsection{Absolute subtraction method}

The results shown here were with the exception of those from Ref. [18] have the combinatoric background subtraction under the assumption that there is a region in $\Delta \phi$ which has no signal (zero yield at minimum-ZYAM). However, the signal distributions are wide in comparison with their distance apart so this ZYAM assumption must result in a lower limit on the jet signal. An alternative method of extracting the jet functions, the absolute subtraction method $[18,22]$ makes no assumption about the shape of the signal. In the case of moderate statistics the two background subtraction 
methods have been shown to agree [9]. However, as seen in Fig. 2.3 with good statistical precision there can be a discrepancy about the shape parameters extracted from the jet functions and the yields extracted under the ZYAM assumption. Also, in the case of poor statistics the absolute background subtraction method provides an improvement over ZYAM because large statistical errors in the correlation functions propagate into large errors on the background level, which lead to large errors on the extracted parameters.

\section{Conclusion}

We have focused on correlations between two hadrons in $\mathrm{Au}+\mathrm{Au}$ collisions at $200 \mathrm{GeV}$. The jet functions in central heavy ion collisions are qualitatively different than in peripheral heavy ion or $\mathrm{p}+\mathrm{p}$ collisions with the addition of the ridge and shoulder structures. Initial results show that the yields, particle ratios, and spectra shape are similar between these two structures. This could indicate that the source of the shoulder and the ridge is similar or that they are sensitive to similar properties of the matter produced in heavy ion collisions. However, further experimental work with high $p_{T}$ triggers and selection of the trigger with respect to the reaction plane needs to be done to determine if the separation of the away side into the shoulder and punch through components is justified. Additionally, results from two and three particle particle correlations at lower energies show a shoulder structure similar to that seen at $200 \mathrm{GeV}$. This challenges the attribution of the shoulder at $200 \mathrm{GeV}$ to a Mach Cone shock-wave. We look forward to further results incorporating more sensitive observables, more particle identification, and more statistics.

\section{References}

1. A. Adare et al. (PHENIX Collaboration), Phys. Rev. C 77, 064907 (2008)

2. S.S. Adler et al. (PHENIX), Phys. Rev. Lett. 91, 172301 (2003). nucl-ex/0305036

3. B. Mohanty et al. (STAR Collaboration). arXiv:0804.4760 [nucl-ex]

4. A. Adare et al., Phys. Rev. C 78, 014901 (2008)

5. C. Chen et al. (PHENIX Collaboration), These proceedings

6. J. Putschke et al. (STAR Collaboration), J. Phys. G S679 (2007)

7. J. Bielcikova, These proceedings

8. E. Wenger et al. (PHOBOS Collaboration). arXiv:0804.3038 [nucl-ex]

9. A. Adare et al. (PHENIX), Phys. Rev. Lett. 98, 232301 (2007)

10. J. Casalderrey-Solana, E.V. Shuryak. hep-ph/0511263

11. B. Abelev et al. (STAR Collaboration). arXiv:0805.0622 [nucl-ex]

12. S. Kniege et al. (CERES Collaboration), J. Phys. G 34, S697-S700 (2007)

13. M. McCumber et al. (PHENIX Collaboration). arXiv:0804.4319 [nucl-ex]

14. M. van Leeuwen et al. (STAR Collaboration). arXiv:0808.4096 [nucl-ex]

15. C. Alt et al. (PHENIX Collaboration), Phys. Rev. Lett. 101, 082301 (2008)

16. R.J. Fries et al., Phys. Rev. Lett. 94, 122301 (2005). nucl-th/ 0407102

17. R.C. Hwa. arXiv:0804.3763 [nucl-th]

18. A. Adare et al. (PHENIX), Phys. Lett. B 649, 359-369 (2007)

19. A.M. Hamed et al. (STAR Collaboration). arXiv:0809.1462 [nucl-ex]

20. A. Adare et al. (PHENIX Collaboration), J. Phys. G 35(10), 104089 (2008)

21. J. Frantz et al. (PHENIX Collaboration), These proceedings

22. A. Sickles et al. (PHENIX Collaboration), Int. J. Mod. Phys. E 16, 3160-3167 (2008). nucl-ex/0702007 